J. Lake Sci. (湖泊科学) , 2016, 28(4): 843-851

DOI 10. 18307/2016. 0418

(c) 2016 by Journal of Lake Sciences

\title{
不同藻类对温度与磷叠加作用的响应模式"
}

\author{
杨 燕 ${ }^{1}$, 朱雪竹 ${ }^{* *}$, 张 民 民 $^{2,3 *}$, 孔繁翔 ${ }^{2,3}$, 黄亚新 ${ }^{4}$ \\ (1: 南京农业大学资源与环境学院,南京 210095) \\ (2: 中国科学院南京地理与湖泊研究所, 湖泊与环境国家重点实验室, 南京 210008) \\ （3: 江苏省区域现代农业与环境保护协同创新中心,淮阴 223300) \\ (4: 南京农业大学生命科学学院, 南京 210095)
}

摘 要: 气候变暖对富营养化引起的蓝藻水华扩张具有叠加作用, 为探索不同藻类对气候变暖与富营养化叠加作用的响 应模式, 选用富营养化水体常见藻类铜绿微囊藻 (Microcystis aeruginosa) 、水华鱼腥藻 (Anabaena flos-aquae) 和斜生栅藻 (Scenedesmus obliquus) 作为材料, 采用室内培养方法, 模拟春末不同升温幅度 $\left(20+3 、 23+3\right.$ 和 $\left.20+6{ }^{\circ} \mathrm{C}\right), 5$ 个磷浓度水平 $(0 、 0.05 、 0.15 、 0.30$ 和 $0.50 \mathrm{mg} / \mathrm{L})$, 通过生长速率、光化学参数等的测定, 分析不同藻类对温度升高和营养盐添加的生长 与光化学响应. 结果表明: 3 种藻的生长速率对温度和磷的叠加作用呈现不同的响应模式: 随着磷浓度的升高, 铜绿微囊 藻的生长速率受温度的叠加作用不断增加, $26^{\circ} \mathrm{C}$ 时差异最大; 水华鱼腥藻生长速率除在 $0.30 \mathrm{mg} / \mathrm{L}$ 磷浓度时受温度叠加 作用比较明显外, 其他磷浓度水平均没有显著的叠加效应; 斜生栅藻在不同磷浓度水平下受温度叠加作用影响有较大波 动, 但无明显差异. 铜绿微囊藻对温度的叠加作用随着磷浓度的增加呈现逻辑斯蒂模式增长, 而水华鱼腥藻和斜生栅藻 均是在特定温度或特定营养盐浓度时呈现最大的叠加作用, 叠加作用的趋势不明显. 从 $F_{\mathrm{v}} / F_{\mathrm{m}}$ 结果可知, 铜绿微囊藻下 降幅度明显高于水华鱼腥藻和斜生栅藻, 随温度升高, 磷浓度越高, 铜绿微囊藻的 $F_{\mathrm{v}} / F_{\mathrm{m}}$ 下降幅度越大, 这可能是铜绿微 囊藻在高生长速率下色素无法积累所致; 水华鱼腥藻的 $F_{\mathrm{v}} / F_{\mathrm{m}}$ 基本保持稳定, 各组间差异较小; 斜生栅藻的 $F_{\mathrm{v}} / F_{\mathrm{m}}$ 在低磷 浓度组明显低于高磷浓度组, 并且随温度升高下降趋势增加, 3 种藻的光学特性响应反映了藻类在叠加作用下生长的变 化. 综上所述, 铜绿微囊澡对温度上升与磷浓度的升高有更强的响应, 导致其产生更高的生长速率, 从而促进了蓝藻水华 的扩张.

关键词: 铜绿微囊藻; 温度;磷;叠加作用;水华鱼腥藻;斜生栅藻

\section{Response patterns of different algae to the interaction between temperature and phos- phorus}

\author{
YANG Yan ${ }^{1}$, ZHU Xuezhu ${ }^{1 * *}$, ZHANG Min ${ }^{2,3 * *}$, KONG Fanxiang ${ }^{2,3}$ \& HUANG Yaxin ${ }^{4}$ \\ (1: College of Resource and Environmental Sciences, Nanjing Agricultural University, Nanjing 210095 , P.R. China) \\ (2: State Key Laboratory of Lake Science and Environment, Nanjing Institute of Geography and Limnology, Chinese Academy \\ of Sciences, Nanjing 210008, P.R.China) \\ (3: Collaborative Innovation Center of Modern Agriculture and Environmental Protection in Jiangsu Province, Huaiyin \\ 223300 , P.R. China) \\ (4: College of Life Sciences, Nanjing Agricultural University, Nanjing 210095, P.R.China)
}

Abstract: Climate warming has a role in promoting cyanobacteria blooms expansion caused by eutrophication. To evaluate the response patterns of different algae on combined effects of climate warming and eutrophication, we simulated different increasing temperature amplitudes in late spring $\left(20+3,23+3,20+6{ }^{\circ} \mathrm{C}\right)$ and five phosphorus concentration levels $(0,0.05,0.15,0.30,0.50$ $\mathrm{mg} / \mathrm{L}$ ) to test the response of the three common algae (Microcystis aeruginosa, Anabaena flos-aquae and Scenedesmus obliquus) in

* 国家自然科学基金项目(31470520，31570457)、江苏省区域现代农业与环境保护协同创新中心项目（HSXT109） 和江苏省太湖水污染治理课题 ( JSZC-G2013-257) 联合资助.2015-08-11 收稿;2015-10-21 收修改稿. 杨燕 (1990 ), 女, 硕士研究生;E-mail:2013103045@ njau.edu.cn.

** 通信作者;E-mail: zhuxuezhu@ njau.edu.cn,E-mail: mzhang@ niglas.ac.cn. 
eutrophic water. The results showed that the growth rate of three algae showed different response patterns. The growth rate of $M$. aeruginosa increased significantly with increased phosphorus concentration and temperature, which suggested that additive effect of temperature and phosphorus increased. The growth rate of A. flos-aquae was subject to temperature in the phosphorus concentration of $0.30 \mathrm{mg} / \mathrm{L}$, with no significant additive effect in the other phosphorus concentration levels. The response in growth rate of S. obliquus presented a great variability at different levels, but no significant difference. With increased phosphorus concentration, the additive effect of temperature and phosphorus on the growth of $M$. aeruginosa showed logistic increasing pattern. The growth of A. flos-aquae and S. obliquus presented the sensitive response to the additive effect at a specific temperature or a specific nutrient concentration, and no significant trend. The photochemical results showed that $F_{\mathrm{v}} / F_{\mathrm{m}}$ of Microcystis declined more significantly than those of A. flos-aquae and $S$. obliquus, which might be due to that the pigment per cell could not be accumulated under this rapid growth rate of Microcystis. The $F_{\mathrm{v}} / F_{\mathrm{m}}$ of $A$. flos-aquae showed weak variability with temperature and phosphorus increasing. The $F_{\mathrm{v}} / F_{\mathrm{m}}$ of $S$. obliquus had a slight decreasing trend with increasing temperature. The dynamics of photochemical characteristics in the three algae were consistent with changes in their growth. In summary, Microcystis had a stronger response to the increased temperature and phosphorus concentration, which led to high growth rates and might contribute to the expansion of cyanobacterial blooms.

Keywords: Microcystis aeruginosa; Anabaena flos-aquae; Scenedesmus obliquus; temperature; phosphorus; additive effect

随着水体富营养化和全球变暖问题的日益加剧, 蓝藻水华不断扩张, 温度与营养盐的叠加作用对蓝藻 水华发生频率及作用程度的影响受到越来越多的关注 ${ }^{[-2]}$. 野外调查研究发现, 在一定的营养盐阈值范围 内, 气候变化可以增加蓝藻占据优势的概率 ${ }^{[3]}$, 并且气候变暖也将增强蓝藻对营养盐的响应程度 ${ }^{[4]}$. Wagner 等 ${ }^{[5]}$ 提出总磷浓度在 70 $215 \mu \mathrm{g} / \mathrm{L}$ 范围内时, 气候变暖能够促进蓝藻占据优势, 王志红等 ${ }^{[6]}$ 也发现硅藻和 绿藻的生长与水温、水体的营养盐程度密切相关, 在中贫以下营养盐条件下, 藻类在低温时更容易生长; 在 中等以上的营养条件下, 高温促进藻类生物量集聚, 更利于形成水华. 并且不同的升温幅度会显著改变蓝藻 水华春季优势确立的时间, 同时这种改变与水体的营养盐水平具有明显的相关性 ${ }^{[7-8]}$. 通常在寡营养水体 中, 富营养化过程对蓝藻水华的扩张具有更大的贡献; 在中营养水体中, 温度升高对蓝藻水华的贡献更大; 在富营养化和超富营养化水体中, 两者展现出显著的叠加作用 ${ }^{[9-11]}$. 另外, 室内多因子的交互试验结果表明, 温度与营养盐浓度、 $\mathrm{pH}$ 值具有叠加效应 ${ }^{[12]}$. 这些研究均确证了富营养化与温度升高对蓝藻水华扩张的叠加 作用.

在我国长江中下游地区, 春季, 尤其是春末, 是蓝藻水华在水体中逐步占据优势的重要时期, 该时期内 温度迅速升高, 升高的温度促进水华蓝藻的繁殖 ${ }^{\left[{ }^{[13]}\right.}$. 水华鱼腥藻 (Anabaena flos-aquae)、铜绿微囊藻 (Microcystis aeruginosa) 是蓝藻水华发生时的主要优势种 ${ }^{[14]}$, 栅藻是春末藻类演替时期的绿藻常见种. 关于温度和 营养盐浓度等环境变量对微囊藻、鱼腥藻、栅藻的影响, 国外已经开展了大量研究. 许多研究人员针对国内 诸多微囊藻、鱼腥藻、㮽澡株系及不同湖泊流域的藻种进行生长特性的研究 ${ }^{[15-16]}$, 甚至开展了多因子交互作 用下生长特性的研究 ${ }^{[17-18]}$. 这些研究通过对微囊藻、鱼腥藻和栅藻的最优生长条件的笁选以及生态位的确 定为蓝藻水华的预测、控制、治理等工作奠定重要的基础, 但是在气候变暖的背景下, 温度升高与富营养化 过程叠加作用对蓝藻水华扩张的定量影响仍有待进一步研究.

本实验选用铜绿微囊藻、水华鱼腥藻、斜生栅藻 ( Scenedesmus obliquus), 通过模拟春季的不同温度与不 同磷浓度的叠加作用, 分析不同条件下 3 种藻的生长和光合特性, 阐述其对温度与磷叠加作用的响应模式, 该研究将有助于深人了解全球变暖过程中温度升高与营养盐叠加作用对蓝藻水华春季优势确立的作用 机制.

\section{1 材料与方法}

\section{1 实验材料}

实验所用铜绿微囊藻、水华鱼腥藻和斜生栅藻均由中国科学院水生生物研究所提供 (藻种编号分别为 FACHB-434、FACHB-245 和 FACHB-14).

\section{2 藻种培养条件}

为探讨温度与营养盐叠加作用对春末蓝藻水华优势形成的影响, 实验温度设置为 $20 、 23$ 和 $26^{\circ} \mathrm{C}$, 以此 
模拟春末不同升温幅度 $\left(20+3 、 23+3\right.$ 和 $\left.20+6^{\circ} \mathrm{C}\right)$, 磷浓度梯度设置为 $0 、 0.05 、 0.15 、 0.30$ 和 $0.50 \mathrm{mg} / \mathrm{L}$, 光暗比 $12 \mathrm{~h}: 12 \mathrm{~h}$, 光照强度 $54 \mu \mathrm{mol} /\left(\mathrm{m}^{2} \cdot \mathrm{s}\right)$, 每组处理设置 3 个重复.

\section{3 实验方法}

取指数生长期 3 种藻 $10 \mathrm{ml}$, 以 5000 转 $/ \mathrm{min}$ 的速度离心 $10 \mathrm{~min}$, 去上清液以去除吸附性磷元素, 再用无 磷的 BG-11 培养基重悬, 离心, 重复此操作 3 次. 最后将除磷后的 3 种藻分别接种到 $0.15 \mathrm{mg} / \mathrm{L}$ 磷浓度的 BG-11 培养基预培养一周 (以 $\mathrm{BG}-11^{[19]}$ 培养基为基础, 通过改变 $\mathrm{K}_{2} \mathrm{HPO}_{4}$ 的浓度配制不同磷浓度的培养基), 再饥饿培养 $48 \mathrm{~h}$.

取一定体积的上述 3 种藻液离心, 重悬, 分别接种到含有 $0 、 0.05 、 0.15 、 0.30 、 0.50 \mathrm{mg} / \mathrm{L}$ 磷浓度的改良 BG-11 培养基中, 其中铜绿微囊藻起始密度为 $1.44 \times 10^{5} \mathrm{cells} / \mathrm{ml}$, 水华鱼腥藻起始密度为 $2.89 \times 10^{5} \mathrm{cell} / \mathrm{s} / \mathrm{ml}$, 斜生栅藻起始密度为 $3.18 \times 10^{5} \mathrm{cells} / \mathrm{ml}$. 实验培养至 3 种藻最先出现生长到平台期 (约 $23 \mathrm{~d}$ 左右), 每隔 $1 \mathrm{~d}$ 取样, 用 Phyto-PAM (ZQ-WALZ004) 测定相对叶绿素 a 浓度 (指示生物生长情况), $F_{\mathrm{v}} / F_{\mathrm{m}}$ (最大光化学量子产 量, 能灵敏反映藻类的生理活性, 是研究藻类营养限制的有力手段), $F_{\mathrm{v}}^{\prime} / F_{\mathrm{m}}^{\prime}$ (有效光化学量子产量, 反映生 物反应中心原初光能捕获效率), 光合利用率 alpha.

\section{4 数据处理}

运用 Excel 2007 和 Origin Pro 8.6 软件进行数据处理及绘图, 应用 SPSS 19 软件对数据进行多重比较、显 著性差异、重复度量等数据分析.

1.4.1 生长速率的计算 根据相对叶绿素 a 浓度, 用 Excel 中的 SLOPE 函数对其作返回经过给定数据点的线 性回归拟合线方程的斜率, 以该值表示本实验中藻类的生长速率, 计算公式为 ${ }^{[20]}: N_{t}=N_{0} \cdot \mathrm{e}^{\mu t}$. 其中, $N_{t}$ 为 $t$ 时 刻浮游植物生物量, $N_{0}$ 为初始浮游植物生物量, $t$ 为倍增时间, $\mu$ 为生长速率.

1.4.2 生长速率差值的计算 生长速率差值是对应磷浓度下生长速率在各温度时的差值, 如: 以 $20^{\circ} \mathrm{C}$ 为基础 增温 $3^{\circ} \mathrm{C}$, 生长速率差值 $\left(20+3^{\circ} \mathrm{C}\right)=$ 生长速率 $\left(23^{\circ} \mathrm{C}\right)-$ 生长速率 $\left(20^{\circ} \mathrm{C}\right)$.

1.4.3 $F_{\mathrm{v}} / F_{\mathrm{m}}$ 下降幅度的计算 下降幅度 $d=\left(x_{n}-x_{1}\right) /\left(t_{n}-t_{1}\right), x_{n}$ 为第 $n$ 天的 $F_{\mathrm{v}} / F_{\mathrm{m}}$ 值, $x_{1}$ 为开始出现下降时 的 $F_{\mathrm{v}} / F_{\mathrm{m}}$ 值, $t_{n}$ 为第 $n$ 天, $t_{1}$ 为开始出现下降的时间.

\section{2 结果}

\section{1 不同温度和磷浓度对 3 种藻生长的影响}

不同温度和磷浓度相互作用下 3 种藻叶绿素 $\mathrm{a}$ 浓度的变化表明, 温度升高导致藻类更快地进人对数生 长期, 达到生物量峰值, 水华鱼腥藻在 $20^{\circ} \mathrm{C}$ 时进入稳定对数生长期并达到生物量峰值, 斜生栅藻在 $23^{\circ} \mathrm{C}$ 时进 人稳定对数生长期并达到生物量峰值, 铜绿微囊藻对数生长期随温度升高不断提前, 在 $26^{\circ} \mathrm{C}$ 时出现生物量 峰值 (图 1). $20^{\circ} \mathrm{C}$ 时, $0.30 \mathrm{mg} / \mathrm{L}$ 以上磷浓度组的铜绿微囊藻叶绿素 a 浓度明显高于其他磷浓度组, 鱼腥藻的 叶绿素 a 浓度在 $0.50 \mathrm{mg} / \mathrm{L}$ 磷浓度组为 $408 \mu \mathrm{g} / \mathrm{L}$, 达到生物量峰值, 斜生栅藻的叶绿素 a 浓度在 $0.15 \mathrm{mg} / \mathrm{L}$ 以上磷浓度组显著增加. $23^{\circ} \mathrm{C}$ 时, $0.30 \mathrm{mg} / \mathrm{L}$ 磷浓度组的铜绿微囊藻叶绿素 $\mathrm{a}$ 浓度依然远高于其他磷浓度 组, 水华鱼腥藻和斜生栅藻在 $0.15 \mathrm{mg} / \mathrm{L}$ 以上磷浓度组时的叶绿素 a 浓度明显增加, 且出现明显差异, 0.50 $\mathrm{mg} / \mathrm{L}$ 磷浓度组斜生栅藻达到生物量峰值, 叶绿素 a 浓度为 $2894.81 \mu \mathrm{g} / \mathrm{L} .26^{\circ} \mathrm{C}$ 时, 3 种藻在 $0.15 \mathrm{mg} / \mathrm{L}$ 以上 磷浓度组生长较快, 受磷浓度影响产生的差异依次减小: 铜绿微囊藻>水华鱼腥藻>斜生栅藻, $0.50 \mathrm{mg} / \mathrm{L}$ 磷浓 度组时铜绿微囊藻叶绿素 a 浓度达到峰值, 为 $4414 \mu \mathrm{g} / \mathrm{L}$.

通过单因素方差分析可见, 3 种藻之间对温度与磷浓度叠加作用的响应存在显著差异 $(P<0.001) .3$ 种 藻对温度、磷浓度以及温度与磷浓度的叠加作用均存在显著的生长响应 $(P<0.001)$. 由各影响因子的偏 $\eta^{2}$ 可知, 时间对 3 种藻的生长影响最大, 温度和时间的交互作用对铜绿微囊藻和水华鱼腥藻影响最小, 斜生栅 藻则受温度影响最小, 而磷浓度对 3 种藻生长的影响远高于温度, 即随持续时间的增加, 营养盐条件对不同 藻类的生长影响较大. 温度和磷浓度对 3 种藻的叠加效果具体表现为: 水华鱼腥藻>铜绿微囊藻>斜生栅藻, 但是对水华鱼腥藻的叠加效果仅体现在个别浓度.

\section{2 不同温度、磷浓度叠加作用对 3 种藻生长速率的影响}

$20^{\circ} \mathrm{C}$ 时, 铜绿微囊藻和水华鱼腥藻在 $0.50 \mathrm{mg} / \mathrm{L}$ 磷浓度组生长速率最快, 是 $0.30 \mathrm{mg} / \mathrm{L}$ 磷浓度组的 2 倍 

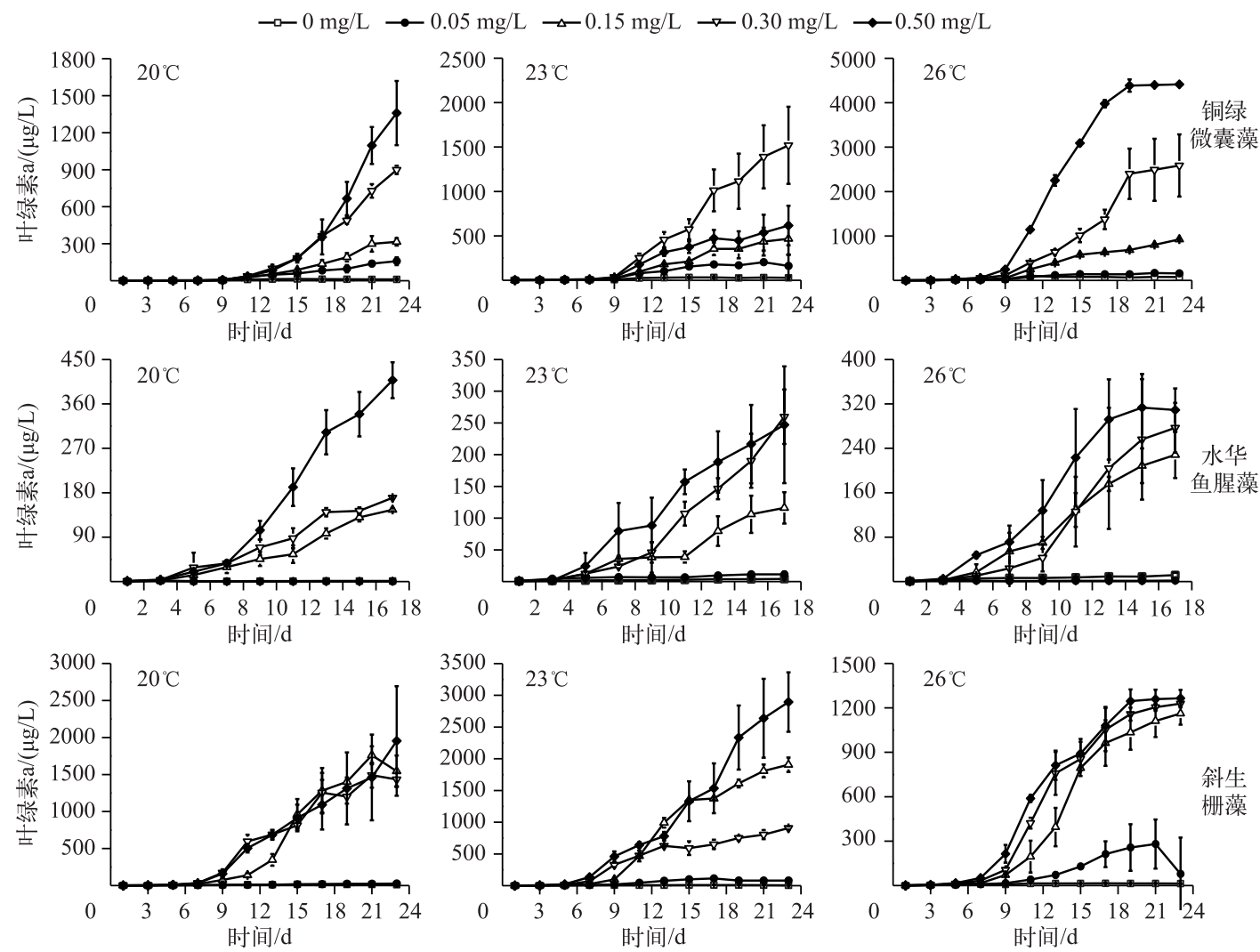

图 1 不同温度、磷浓度叠加作用下 3 种藻叶绿素 a 浓度的变化

Fig. 1 The changes of chlorophyll-a concentrations of three algae under the additive effect of different temperatures and phosphorus concentrations

左右, 斜生栅藻因磷浓度不同而产生的生长速率之间的差异较不明显; $23^{\circ} \mathrm{C}$ 时, 3 种藻均是在高磷浓度组生 长速率较快, 铜绿微囊藻生长速率随磷浓度增加先增加后下降, 水华鱼腥藻保持增加趋势, 而斜生栅藻没有 显著变化规律; $26^{\circ} \mathrm{C}$ 时, 铜绿微囊藻在各磷浓度组间的差异显著 $(P<0.05)$, 但水华鱼腥藻和斜生栅藻在高磷 浓度组无明显差异 (图 2).

生长速率差值反映了不同的增温幅度对藻类生长快慢的影响 (图 3), 当温度由 $20^{\circ} \mathrm{C}$ 升高 $3^{\circ} \mathrm{C}$ 后, 铜绿微 囊藻的生长速率差值曲线为开口向下的抛物线状, 水华鱼腥藻和斜生柾藻的生长速率差值曲线无明显规 律; 当温度由 $23^{\circ} \mathrm{C}$ 升高 $3^{\circ} \mathrm{C}$ 后, 各磷浓度组铜绿微囊藻生长速率差值的增长均优于 $20^{\circ} \mathrm{C}$ 增温 $3^{\circ} \mathrm{C}$, 水华鱼腥 藻生长速率差值较 $20^{\circ} \mathrm{C}$ 增温 $3^{\circ} \mathrm{C}$ 条件下的差值变化快, 斜生柾藻在 $0.30 \mathrm{mg} / \mathrm{L}$ 磷浓度组生长速率差值增长 最快, 此时, 铜绿微囊藻的生长速率差值曲线呈现逻辑斯蒂模式, 水华鱼腥藻和斜生栅藻的生长速率差值曲 线无明显规律; 当温度由 $20^{\circ} \mathrm{C}$ 升高 $6^{\circ} \mathrm{C}$ 后, 铜绿微囊藻随着磷浓度的升高生长速率差值增长加快, 水华鱼腥 藻在 $0.30 \mathrm{mg} / \mathrm{L}$ 磷浓度组生长速率差值增加最快, 斜生栅藻的生长呈衰亡趋势, 此时, 铜绿微囊藻的生长速 率差值曲线呈现逻辑斯蒂模式, 水华鱼腥藻和斜生栅藻的生长速率差值曲线无明显规律.

\section{3 不同温度、磷浓度叠加作用对 3 种藻 $F_{\mathrm{v}} / F_{\mathrm{m}}$ 的影响}

$F_{\mathrm{v}} / F_{\mathrm{m}}$ 在一定程度上可以反映藻类生长的最大光合作用能力. 随着温度升高, 铜绿微囊藻的最大光化学 量子产量 $F_{\mathrm{v}} / F_{\mathrm{m}}$ 的下降时间节点不断提前, $F_{\mathrm{v}} / F_{\mathrm{m}}$ 总体表现为先迅速升高, 最高为 0.56 , 再缓慢下降至 $0.1 \sim$ 0.2 后逐渐保持稳定, 高磷浓度组的 $F_{\mathrm{v}} / F_{\mathrm{m}}$ 受温度抑制明显; 水华鱼腥藻的 $F_{\mathrm{v}} / F_{\mathrm{m}}$ 在温度与磷浓度叠加作用 下,基本保持稳定, 各组间差异不显著, 其 $F_{\mathrm{v}} / F_{\mathrm{m}}$ 最高为 0.36 ; 斜生栅藻的 $F_{\mathrm{v}} / F_{\mathrm{m}}$ 在低磷浓度组明显低于高磷 

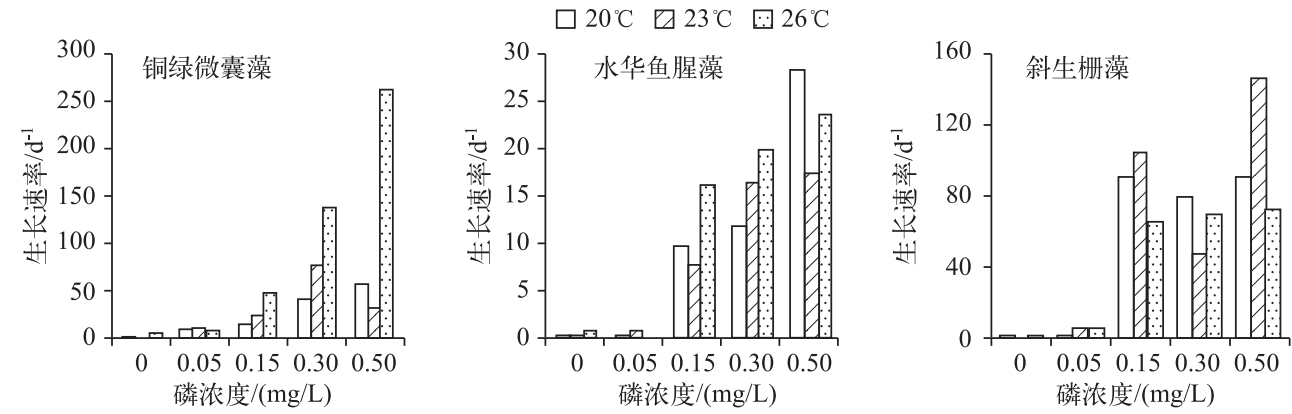

图 2 不同温度、磷浓度叠加作用下 3 种藻生长速率比较

Fig.2 The comparison of growth rate of the three algae under the additive effect of different temperatures and phosphorus concentrations
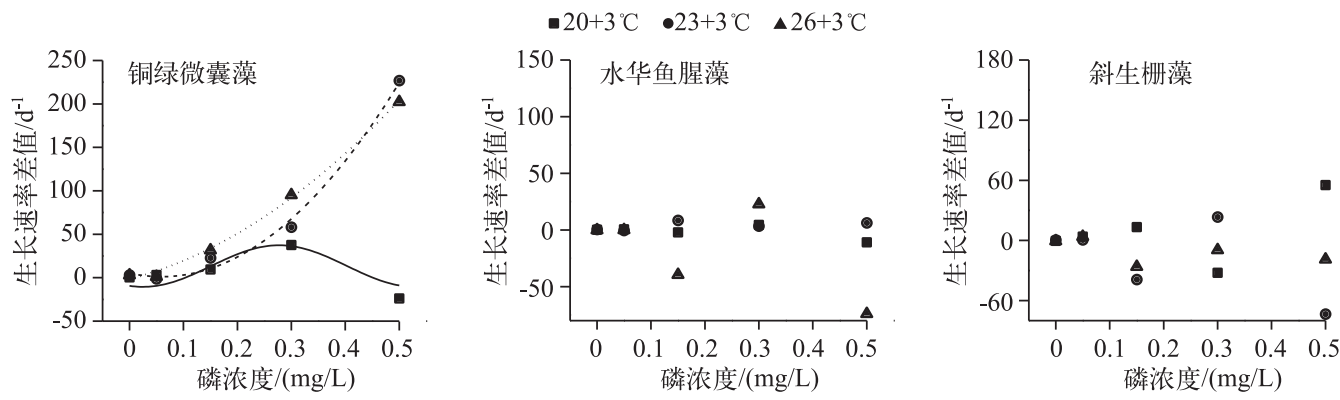

图 3 不同温度增幅、磷浓度叠加作用下 3 种藻生长的比较

Fig. 3 The comparison of growth difference of three algae under the additive effect of different increased temperatures and phosphorus concentrations

浓度组, 并且随温度升高下降的趋势增加 (图 4). 3 种藻的有效光化学量子产量 $F_{\mathrm{v}}{ }^{\prime} / F_{\mathrm{m}}{ }^{\prime}$ 、光合利用率 alpha 变 化结果与 $F_{\mathrm{v}} / F_{\mathrm{m}}$ 相似.

\section{3 讨论}

春季形成蓝藻水华受多种因素的影响, 湖泊富营养化是主要原因 ${ }^{[21-22]}$, 而全球气候变暖被认为是加剧 蓝藻水华的重要因素之一 ${ }^{[23]}$, 两者间具有明显的叠加效应, 并且不同藻类对这种叠加作用的响应方式不 同 ${ }^{[24-25]}$. 本研究发现, 铜绿微囊藻、水华鱼腥藻和斜生栅藻生长速率对温度和磷的叠加作用呈现不同的响应 模式: 铜绿微囊藻对温度的叠加作用随着磷浓度的增加而呈逻辑蒂斯模式增长; 水华鱼腥藻生长速率除在 $0.30 \mathrm{mg} / \mathrm{L}$ 时受温度叠加作用比较明显外, 其他磷浓度水平均没有显著的叠加效应; 斜生柾藻在不同磷浓度 水平下受温度叠加作用影响有较大波动,但无明显差异.

气候变暖使得富营养化的淡水湖泊中, 微囊藻的分布范围变广且数量不断增加 ${ }^{[26]}$. 王亚超等 ${ }^{[27]}$ 研究发 现在温度高于 $20^{\circ} \mathrm{C}$ 时有利于微囊藻生长, 并随着磷浓度增加而增加. 室内多因子的模拟实验结果也表明, 气 候因子与营养盐浓度具有叠加效应, 升高温度和添加磷对有毒微囊澡生长速率的促进程度远高于单因子实 验组 ${ }^{[28]}$. 本实验中随着磷浓度的升高, 铜绿微囊藻的生长速率受温度的叠加作用不断增加, $26^{\circ} \mathrm{C}$ 时差异最 大, 并且呈现逻辑斯蒂增长模式, 这在确证以前研究结果的同时, 进一步揭示了其随温度和磷浓度增加的变 化趋势. Rigosi 等 ${ }^{[29]}$ 研究发现高磷条件下, 藻类生长受温度影响明显, 低磷条件下, 藻类生长则受营养盐影 响比较明显, 而微囊藻的生长过程对温度更敏感. 本研究发现铜绿微囊藻不仅对温度的升高敏感, 其对营养 盐的增加同样敏感,这可能是其能够对两者叠加作用表现出逻辑斯蒂响应模式的重要原因. 

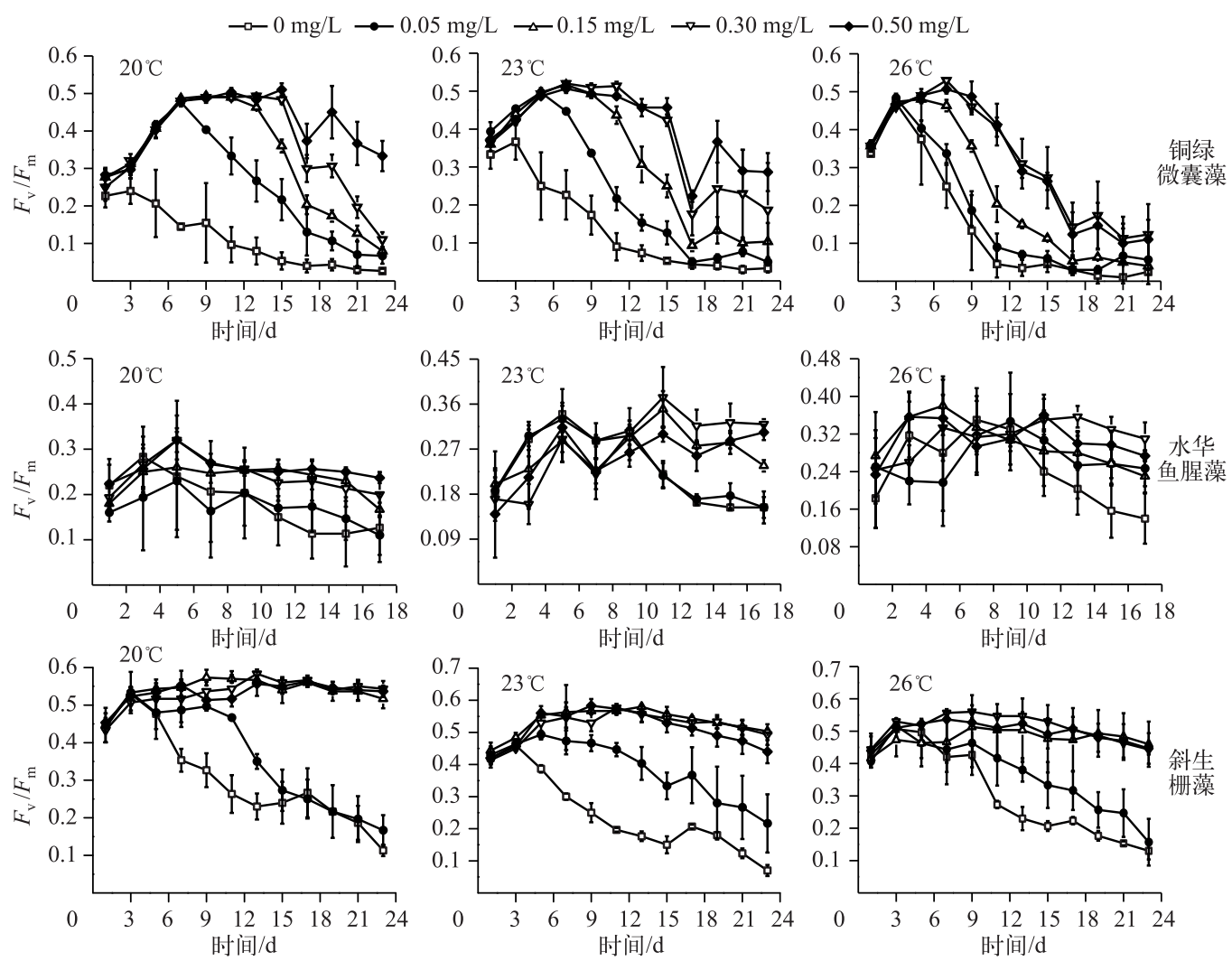

图 4 不同温度、磷浓度叠加作用下 3 种藻类的 $F_{\mathrm{v}} / F_{\mathrm{m}}$ 的变化

Fig.4 The changes of $F_{\mathrm{v}} / F_{\mathrm{m}}$ of the three algae under the additive

effect of different temperatures and phosphorus concentrations

水华鱼腥藻比较能够适应低温环境, 常锋毅等 ${ }^{[30]}$ 的研究显示鱼腥藻的最适生长温度在 $25^{\circ} \mathrm{C}$ 左右, 温度 升高导致其生长速率明显下降, 而且随着磷浓度的增加其生长速率明显加快. 顾启华等 ${ }^{[31]}$ 研究也发现鱼腥 藻在高磷浓度时具有明显的生长优势, 低磷是鱼腥藻生长的限制因子. 由此可见, 鱼腥藻对于温度的增加并 不像铜绿微囊藻那样在较大范围内都呈现正响应, 特别是在春末, 气候变暖带来的温度升高可能并不会促 进其扩张, 反而导致峰值的过早消退. 与温度相反, 其对营养盐增加的响应则更为敏感, 富营养化的加剧可 能会促进其进一步扩张.

斜生栅藻是绿藻水华的常见种, 是春季蓝藻水华形成过程中处于竞争地位的藻类之一 ${ }^{[32]}$. 郑忠明等 ${ }^{[33]}$ 研究发现栅藻在低温 $\left(22^{\circ} \mathrm{C}\right)$ 时生长速率及生物量达到最大, 随着温度的增加其生长速率逐渐降低, 但是降 低的幅度并不显著, 因此可见栅藻对于温度的响应并不敏感. 栅藻对磷的加富过程具有明显的响应, 随着磷 浓度的增加其生长速率明显加快 ${ }^{[34]}$. 万蕾等 ${ }^{[35]}$ 研究也发现, 在贫营养条件下, 栅藻和微囊藻能够互相促进 生长, 在富营养水体中, 两种藻的竞争受氮磷比的影响, 而在超富营养化水体中, 栅藻容易形成优势. 但是当 温度升高与磷浓度增加叠加起作用时, 特别是在本研究的温度范围内, 温度升高使得其生长速率降低, 而磷 浓度升高导致生长速率增加,两者叠加可能是导致本研究中栅藻没有显著响应的原因.

光合作用是藻类生长发育的能量基础, 藻类在进行光合作用时, 其光能一部分用来进行光化学反应, 推 动光量子能量传递转换, 一部分以热的形式进行非辐射能量耗散, 还有一部分则以叶绿素苂光的形式进行 辐射能量耗散. 利用藻类叶绿素荧光则能够反映出其光合作用能力的变化 ${ }^{[36-37]}$. 本实验研究发现, 随温度升 高, 铜绿微囊藻 $F_{\mathrm{v}} / F_{\mathrm{m}}$ 明显降低, 斜生栅藻的下降幅度较小, 而水华鱼腥藻无明显的变化规律, 这可能是由于 
高温、高磷浓度条件下藻类快速分裂繁殖, 导致单个细胞的色素无法积累, 使得单个细胞的最大光合作用能 力显著减弱所致 ${ }^{[38]} .3$ 种藻类光合特性的这种变化也印证了其对温度与磷叠加作用的响应趋势.

由此可见, 水华鱼腥藻和斜生栅藻可能对营养盐的增加更为敏感, 而铜绿微囊藻可能对温度的升高更 为敏感. 在富营养化或超富营养化湖泊中, 铜绿微囊藻水华对于气候变暖的叠加作用将有更强的响应, 因 此,气候变暖可能对于富营养化湖泊中的铜绿微囊藻水华扩张有更大的促进作用.

气候变暖和富营养化的叠加作用促进春末蓝藻水华的加剧, 基于铜绿微囊藻对这种叠加作用的强烈响 应, 在未来应对蓝藻水华问题的工作中, 应区分引起蓝藻水华的藻类的种类, 并更加有针对性的提出关于铜 绿微囊藻水华的应对措施. 同时, 在这个叠加作用中, 营养盐的变化起到更加基础的作用 ${ }^{[39]}$, 而营养盐作为 可控因素, 在气候变暖的条件下, 蓝藻水华的控制亟需设定更低的营养盐國值, 只有更为严格的控制营养盐 才能减缓甚至抵消气候变暖对富营养化的叠加作用.

\section{4 参考文献}

[ 1 ] Huber V, Wagner C, Gerten D et al. To bloom or not to bloom: contrasting responses of cyanobacteria to recent heat waves explained by critical thresholds of abiotic drivers. Oecologia, 2012, 169: 245-256.

[ 2 ] Joohnk KD, Huisman J, Sharples J et al. Summer heatwaves promote blooms of harmful cyanobacteria. Global Change Biology, 2008, 14: 495-512.

[ 3 ] Yin Zhikun, Li Zhe, Wang Sheng et al. Effect of light and temperature on growth kinetics of Anabaena flos-aquae under phosphorus limitation. Environmetal Science, 2015, 36(3) : 963-968 (in Chinese with English abstract). DOI 10.13227/J. HJKX.2015.03.027. [殷志坤, 李哲, 王胜等. 磷限制下光照和温度对水华鱼腥藻生长动力学的影响. 环境科学, $2015,36(3)$ : 963-968. ]

[ 4 ] Zhu Wei, Wan Lei, Zhao Lianfang. Interspecies competition rule of algae under different temperature and nutrient concentration condition. Ecology and Environment, 2008, 17 (1): 6-11 (in Chinese with English abstract). DOI 10.3969/J. ISSN.1674-5906.2008.01.002. [ 朱伟, 万蕾, 赵联芳. 不同温度和营养盐质量浓度条件下藻类的种间竞争规律. 生 态环境, 2008, 17(1): 6-11.]

[ 5 ] Wagner C, Adrian R, Williamson CE et al. Cyanobacteria dominance: quantifying the effects of climate change. Limnology and Oceanography, 2009, 54(6): 2460-2468.

[ 6 ] Wang Zhihong, Cui Fuyi. Influence of water temperature and trophic value on algae blooming in reservoirs. Ecology and Environment, 2005, 14(1) : 10-15 (in Chinese with English abstract). DOI 10.3969/J. ISSN.1674-5906.2005.01.003. [王志红, 崔福义. 水温与营养值对水库藻华态势的影响. 生态环境, 2005, 14(1): 10-15. ]

[ 7 ] Deng J, Qin B, Paerl HW et al. Earlier and warmer springs increase cyanobacterial ( Microcystis spp. ) blooms in subtropical Lake Taihu, China. Freshwater Biology, 2014, 59(5) : 1076-1085. DOI 10.1111/FWB.12330.

[ 8 ] Zhang M, Duan H, Shi X et al. Contributions of meteorology to the phenology of cyanobacterial blooms: implications for future climate change. Water Research, 2012, 46(2) : 442-452. DOI 10.1016/J.WATRES.2011.11.013.

[ 9 ] Wang J, Chen J, Meng S. The effects of environmental factors on the growth and competition of algae. Asian Agricultural Research, 2013, 29(17) : 52-56.

[10] Sotero-Santos RB, Carvalho EG, Dellamano-Oliveira MJ et al. Occurrence and toxicity of an Anabaena bloom in a tropical reservoir (Southeast Brazil). Harmful Algae, 2008, 7: 590-598. DOI 10.1016/J.HAL.2007.12.017.

[11] Wang Jing, Qiu Liping, Meng Shunlong et al. Influences of nitrogen-phosphorus ratio on the growth and competition of Chlorella vulga and Anabaena sp. strain pcc. Agricultural Science \& Technology, 2014, 42(5) : 316-320(in Chinese with English abstract). DOI 10.3969/J.ISSN.1009-4229.2015.08.042. [王菁, 表丽萍, 孟顺龙等. 氮磷比对鱼腥藻和普通 小球藻生长竞争的影响. 江苏农业科学, 2014, 42(5) : 316-320.]

[12] Yuan Lina, Song Wei, Xiao Lin et al. The overall orthogonal desistudy of multifactor interaction on the growth of Microcystis aeruginosa in the presence of adnascent pseudomonas. Journal of Nanjing University: Natural Sciences, 2008, 44(4) : 408-414 (in Chinese with English abstract). DOI 10.3321/J.ISSN :0469-5097.2008.04.011. [袁丽娜, 宋炜, 肖琳等. 多 环境因素全面正交作用对铜绿微囊藻生长的效应研究. 南京大学学报: 自然科学版, 2008, 44(4) : 408-414.]

[13] Kong Fanxiang, Gao Guang. Hypothesis on cyanobacteria bloom-forming mechanism in large shallow eutrophic lakes. Acta Ecologica Sinica, 2005, 25(3) : 589-595 (in Chinese with English abstract). DOI 10.3321/j.issn: 1000-0933.2005.03. 
028. [ 孔繁翔, 高光. 大型浅水富营养化湖泊中蓝藻水华形成机理的思考. 生态学报, 2005, 25(3): 589-595.]

[14] Wang Liang, Dong Le, Chen Hanliang et al. Study on water-bloom of Microcystis in ponds. Journal of Inner Mongol University For Nationalities: Natural Sciences, 2002, 17(1) : 45-47(in Chinese with English abstract). DOI 10.3969/ J.ISSN. 1671-0185.2002.01.015. [王亮, 董乐, 程汉良等. 池塘微囊藻水华的研究. 内蒙古民族大学学报: 自然科学版, $2002,17(1): 45-47$.

[15] Brookes JD, Carey CC. Resilience to Blooms. Science, 2011, 334(6052) : 46-47. DOI 10.1126/SCINENCE 1207349.

[16] Martinez M, Jimenez J, Yousfi F. Influence of phosphorus concentration and temperature on growth and phosphorus uptake by the microalga Scenedesmus obliquus. Bioresource Technology, 1999, 67(3) : 233-240.

[17] Xie Lijuan, Miao Hengfeng Yan Qun et al. Effects of phosphorus limitation to the growth of Microcystis aeruginosa and Microcystis flos-aquae. Shanghai Environmental Sciences, 2011, 30(3): 124-129(in Chinese with English abstract). [谢利] 娟, 缪恒锋, 严群等. 磷限制对铜绿和水华微囊藻生长状况的影响. 上海环境科学, 2011, 30(3): 124-129.]

[18] Xue Lingzhan, Chen Xiaochen, Huang Zhongchi et al. Interactive effects of temperature and phosphorus on the growth of Microcystis aeruginosa and Chlorella vulgaris. Anhui Agricultural Science Bulletin, 2011, 17(13): 23-25(in Chinese with English abstract). DOI 10.3969/J.ISSN.1007-7731.2011.13.010. [薛凌展, 陈小晨, 黄种持等. 温度和磷交互作用对 铜绿微囊藻和小球藻生长的影响. 安徽农学通报, 2011, 17(13): 23-25.]

[19] Hu Xiaozhen, Ma Zuyou, Yi Wenli et al. Growth of Microcystis aeruginosa and Scendesmus quadricauda in four different mediums. Reserch of Environmental Science, 2004, 17(z1): 55-57 (in Chinese with English abstract). DOI 10.3321/J. ISSN : 1001-6929.2004.z1.013. [胡小贞, 马祖友, 易文利等. 4 种不同培养基下铜绿微囊藻和四尾栅藻生长比较. 环境科学研究, 2004, 17(z1): 55-57.]

[20] Sun Jun, Ning Xiuren. Marine phytoplankton specific growth rate. Advances in Earth Science, 2005, 20(9): 939-945(in Chinese with English abstract). DOI 10.3321/J.ISSN : 1001-8166.2005.09.003. [孙军, 宁修仁. 海洋浮游植物群落的 比生长率. 地球科学进展, 2005, 20(9): 939-945.]

[21] Spears BM, Carvalho L, Dudley B et al. Variation in chlorophyll a to total phosphorus ratio across 94 UK and Irish lakes: Implications for lake management. Journal of Environmental Management, 2013 , 115(3) : 287-294. DOI 10.1016/J.JENVMAN.2012.10.011.

[22] Conley DJ. Controlling eutrophication: nitrogen and phosphorus. Science, 2009, 323(5917) : 1014-1015. DOI 10.1126/ SCIENCE. 1167755 .

[23] Paerl HW, Huisman J. Climate -Blooms like it hot. Science, 2008, 320(5872) : 57-58. DOI 10.1126/SCIENCE.1155398.

[24] Taranu ZE, Zurawell RW, Pick F et al. Predicting cyanobacterial dynamics in the face of global change: the importance of scale and environmental context. Global Change Biology, 2012, 18(12) : 3477-3490. DOI 10.1111/GCB.12015.

[25] Anneville O, Gammeter S, Straile D. Phosphorus decrease and climate variability: mediators of synchrony in phytoplankton changes among European peri-alpine lakes. Freshwater Biology, 2005, 50(10) : 1731-1746. DOI 10.1111/J.1365-2427. 2005.01429.

[26] Cao HS, Tao Y, Kong FX et al. Relationship between temperature and cyanobacterial recruitment from sediments in laboratory and field studies. Journal of Freshwater Ecology, 2008, 23(3) : 405-412.

[27] Wang Yachao, Xu Hengsheng, Wang Guoxiang et al. The impact of nitrogen, phosphorus and other environmental factors on Microcystis and Anabaena growth in Taihu Lake. Environmental Monitoring and Forewarning, 2013, 5(1): 7-10(in Chinese with English abstract). DOI 10.3969/J.ISSN.1674-6732.2013.01.002. [王亚超, 徐恒省, 王国祥等. 氮、磷等 环境因子对太湖微囊藻与水华鱼腥藻生长的影响. 环境监控与预警, 2013, 5( 1) : 7-10. DOI 10.3969/J.ISSN.16746732.2013.01.002.

[28] Davis TW, Berry DL, Boyer GL et al. During cyanobacteria blooms. Harmful Algae, 2009, 8( 5) : 715-725. DOI 10. 1016/J.HAL.2009.02.004.

[29] Rigosi A, Carey CC, Ibelings BW et al. The interaction between climate warming and eutrophication to promote cyanobacteria is dependent on trophic state and varies among taxa. Limnology and Oceanography, 2014, 59(1) : 99-114. DOI 10. 4319/LO.2014.59.1.0099.

[30 Chang Fengyi, Pan Xiaojie, Kang Lijuan et al. A study on the growth and some physiological characteristics of Anabaean spiroides isolated from Lake Erhai. Acta Hydrobiological Sinica, 2009, 33 (3) : 385-390 (in Chinese with English abstract). DOI 10.3724/SP.J.0000.2009.30385. [ 常锋毅, 潘晓洁, 康丽娟等. 洱海螺旋鱼腥藻生长生理特性的初步 
研究. 水生生物学报, 2009, 33(3) : 385-390.]

[31] Gu Qihua, Zhao Lin, Tan Xin. Studies on conpetition predominance of Microcystis aeruginosa, Anabaena spiroides and Aphanizomeno flos-aquae. Journal of Anhui Agricultural Sciences, 2007, 35(7) : 1990-1991, 2031 (in Chinese with English abstract). DOI 10.3969/j.issn.0517-6611.2007.07.068. [顾启华, 赵林, 谭欣. 铜绿微囊藻、螺旋鱼腥藻和水华束 丝藻竞争优势的研究. 安徽农业科学, 2007, 35(7)：1990-1991, 2031.]

[32] Chen Dehui, Liu Yongding, Yuan Junfeng et al. Experiments of mixed culture and calculation of competitive parameters between Microcystis cyanobacteria and Scenedesmus. Acta Ecologica Sinica, 1999, 19(6) : 908-913( in Chinese with English abstract). DOI 10.3321/J.ISSN:1000-0933.1999.06.025. [ 陈德辉, 刘永定, 袁峻峰等. 微囊藻和栅藻共培养实 验及其竞争参数的计算. 生态学报, 1999, 19(6) : 908-913.]

[33] Zhen Zhongming, Bai Peifeng, Lu Kaihong et al. Growth characteristics and competitive parameters of Microcystis aeruginosa and Scendesmus quadrecauda at different temperatures. Acta Hydrobiological Sinica, 2008, 32(5) : 720-728(in Chinese with English abstract). DOI 10.3724/sp.j.0000.2008.50720. [郑忠明, 白培峰, 陆开宏等. 铜绿微囊藻和四尾栅 藻在不同温度下的生长特性及竞争参数计算. 水生生物学报, 2008, 32( 5) : 720-728. ]

[34] Xu Hai, Yang Linzhang, Mao Hua et al. Dynamic studies on the effect of phosphorus on the growth of Microcystis aeruginosa and Scendesmus obliquus. Ecology and Environment, 2006, 15(5): 921-924(in Chinese with English abstract). DOI 10.3969/J.ISSN.1674-5906.2006.05.006. [许海, 杨林章, 茅华等. 铜绿微囊藻、斜生栅藻生长的磷营养动力学特 征. 生态环境, 2006, 15(5): 921-924.

[35] Wan Lei, Zhu Wei, Zhao Lianfang. Effect of nitrogen and phosphorus on growth and competition of M. aeruginosa and $S$. quadricauda. Environmental Science, 2007, 28(6) : 1230-1235(in Chinese with English abstract). DOI 10.3321/J.ISSN : 0250-3301.2007.06.011. [万蕾, 朱伟, 赵联芳. 氮磷对微囊藻和栅藻生长及竞争的影响. 环境科学, 2007, 28(6): 1230-1235. ]

[36] Consalvey M, Perkins RG, Paterson DM et al. Pam fluorescence: a beginners guide for benthic diatomists. Diatom Research, 2005, 20(1): 1-22.

[37] Maxwell K, Johnson GN. Chlorophyll fluorescence-a practical guide. Journal of Experimental Botany, 2000, 51(345): 659-668.

[ 38 ] Schitüter L, Bo R, Søndergaard M. Nutrient limitation in relation to phytoplankton carotenoid/chlorophyll a ratios in freshwater mesocosms. Journal of Plankton Research, 1997, 19(7) : 891-906. DOI 10.1093/PLANKT/19.7.891.

[39] Davis TW, Berry DL, Boyer GL et al. The effects of temperature and nutrients on the growth and dynamics of toxic and non-toxic strains of Microcystis during cyanobacteria blooms. Harmful Algae, 2009, 8( 5) : 715-725. DOI 10.1016/J.HAL. 2009.02.004. 